\title{
Overexpression of MicroRNA-340-5p Attenuates Lung Cancer Cell Proliferation and Invasion through Wnt/ Beta-Catenin Signaling Pathway
}

\author{
L. S. CHEN, Z. G. XIE, Z. G. WU AND Z. Z. CHEN*
}

Department of Emergency, The Second Affiliated Hospital of Fujian Medical University, No. 34, Zhongshan North Road, Quanzhou, Fujian 362000, China

Chen et al.: Overexpression of MicroRNA-340-5p Attenuates Lung Cancer Cells

\begin{abstract}
Numerous studies have screened and dysregulated the microRNA in various cancers via microarray assay. In this dysregulated microRNA, microRNA-340-5p attracted our attention due to its high conservation and fold change. MicroRNA-340-5p is abnormally expressed in several cancers and plays an important role. However, the expression and function of microRNA-340-5p in lung cancer are still unknown. Our aim was to explore the role of microRNA-340-5p in lung cancer and its underlying molecular mechanism. The lung cancer cell lines of human lung squamous cell carcinoma cell line SKMES1, human lung adenocarcinoma cell line A549, lung adenocarcinoma cells H23, human lung cancer cell line SPC-A1 and human squamous cell carcinoma $\mathrm{H520}$ were used in present study. Real time polymerase chain reaction was performed to detect microRNA-340-5p and beta-catenin messenger RNA expression. Transwell assay was conducted to determine the invasion of human squamous cell carcinoma $\mathbf{H 5 2 0}$ cells under different treatment conditions. Our study showed that the expression of microRNA-340-5p in lung cancer tissues and was significantly lower than normal tissues. Moreover, overexpression of microRNA-340-5p could suppress the invasion and proliferation of lung cancer cells. We also confirmed that microRNA-340-5p and beta-catenin are negatively correlated in lung cancer tissues and the role of microRNA-340-5p in lung cancer can be reversed by overexpression of beta-catenin. Our results demonstrate that up-regulation of microRNA-340-5p attenuates lung cancer cell invasion and proliferation via inhibiting wingless-related integration site/beta-catenin pathway. The data will provide new insights for the treatment of lung cancer and new guidance for clinical treatment of lung cancer.
\end{abstract}

Key words: MicroRNA-340-5p, lung cancer, wingless-related integration site/ $\beta$-catenin signaling pathway

Lung cancer is the most common cancer and the first leading cause worldwide ${ }^{[1]}$. Many patients are in advanced stage when they are diagnosed with lung cancer and about $30 \%$ of early lung cancer patients have distant metastasis ${ }^{[2]}$. Lung cancer has become a lifethreatening disease that seriously affects the patients quality of life ${ }^{[3]}$. Therefore, a thorough understanding of its mechanism can provide a new perspective for the diagnosis and treatment of lung cancer.

The development factors of lung cancer include invasion, migration, abnormal proliferation and angiogenesis ${ }^{[4]}$. Invasion is an important process in which tumors invade normal tissues and deteriorate disease ${ }^{[5]}$. Although many studies are exploring the potential lung cancer cells invasion mechanism, the potential mechanism and function of lung cancer cell invasion are not fully understood.

Micro ribonucleic acid (miRNA) is a group of singlestranded non-coding regulatory RNA molecules with a length of 21-23 nucleotides. It is highly conservative and has many biological functions ${ }^{[6]}$. Studies have shown that microRNAs have dual biological functions of

*Address for correspondence

E-mail: bbp852369@163.com 
promoting or suppressing cancer and play an important role in the invasion and proliferation of tumors ${ }^{[7,8]}$.

In recent $10 \mathrm{y}$, miRNAs have become a new research hotspot in many diseases ${ }^{[9]}$. MiRNAs are abundantly expressed in human tissues and blood and are suitable for the diagnosis and treatment of lung cancer as candidate molecular markers and targets ${ }^{[10]}$. However, the association between microRNA-340$5 p$ (miR-340-5p) and lung cancer remains unclear. There is no doubt that investigating the effect and mechanism of miR-340-5p in the pathophysiology of lung cancer will deepen the understanding of lung cancer progression.

Some studies have shown expression of miR$340-5 p$ was significantly down-regulated in some cancers and exert a crucial role in these cancers ${ }^{[11,12]}$. In present study, we conducted real-time reverse transcription-polymerase chain reaction (RT-PCR) analysis to examine the expression of miR-340-5p in lung cancer tissues and to explore the potential function in lung cancer cell line. We found that overexpression of miR-340-5p could suppress the invasion and proliferation of lung cancer cells. In addition, we also demonstrate that wingless-related integration site $(\mathrm{Wnt}) / \beta$-catenin pathway is involved in the inhibitory role of miR-340-5p in lung cancer cells. Taken together, we elucidated a novel miRNA with unknown function in lung cancer and studied its role and mechanism in lung cancer. Our study will provide new insights into the treatment of lung cancer and new clues for clinical treatment.

\section{MATERIALS AND METHODS}

\section{Tissue samples and cell culture:}

Cancer tissues and adjacent normal tissues were obtained from 38 patients with lung cancer undergoing resection between May 2017 and October 2018 in the second affiliated hospital of Fujian medical university. All tissues were confirmed by pathological examination. Informed consent was obtained from all patients and ethical approval was obtained from the second affiliated hospital of Fujian medical university.

The cell lines of human bronchial epithelial cell line (16HBE), human lung squamous cell carcinoma cell line (SKMES1), human lung adenocarcinoma cell line (A549), lung adenocarcinoma cells (H23), human lung cancer cell line (SPC-A1) and human squamous cell carcinoma (H520) used in this study were obtained from cell center of Shanghai academy of life sciences (Shanghai, China). Cells were incubated in Dulbecco's modified eagle medium (DMEM) (Thermo Fisher Scientific, USA) containing $10 \%$ fetal bovine serum (FBS) (Life Technologies, Grand Island, USA) and were cultured in a humidified atmosphere at $37^{\circ}$ with $5 \% \mathrm{CO}_{2}$.

RNA quantification and quantitative reverse transcription polymerase chain reaction (qRTPCR) assay:

Total RNA of lung cancer and normal tissues and lung cancer cell lines were extracted using Trizol following the manufacturer's instructions. After total RNA was retrovirally transcribed into complementary deoxyribonucleic acid (cDNA), the amplification of cDNA was performed by Platinum SYBR Green RT-qPCR SuperMix-UDG reagents (Invitrogen, Carlsbad, CA) and the MX3000P system (Stratagene).

The expression of miR-340-5p and $\beta$-catenin was normalized and quantified in lung cancer cell lines using glyceraldehyde 3-phosphate dehydrogenase (GAPDH) as internal reference. Concrete steps were denaturated at $95^{\circ}$ for $15 \mathrm{~s}$, then 35 cycles at $95^{\circ}$ for $10 \mathrm{~s}, 58^{\circ}$ for $15 \mathrm{~s}$ and $60^{\circ}$ for $20 \mathrm{~s}$. miR-340-5p and $\beta$-catenin expression fold change was calculated by the $2^{-\Delta \Delta C t}$ method according to normalization to GAPDH. Primers are provided in Table 1.

TABLE 1: SEQUENCES OF PRIMERS FOR PCR

\begin{tabular}{ccc}
\hline Genes & Forward primer (5'-3') & Reverse primer (5'-3') \\
\hline GAPDH & AAGGGCCGTCCCGGTACGA & GCAATATACCCGCTGCAGT \\
miR-340-5p & TGGCGTTCCCTTTAGGCTA & AAGGTGCCCTGTGTTATCC \\
B-catenin & ACTAAGCCCACTGGTTAG & GGCTCGGTATTTCCCCGGT \\
\hline
\end{tabular}




\section{Plasmid transfection:}

H520 cells were transfected with $50 \mathrm{nM}$ miR-340$5 \mathrm{p}$ mimics (Genescript, Nanjing, China). For the overexpression experiments, the transfection efficiency was verified in infected H520 cells by qRT-PCR. Lipofectamine 2000 (Invitrogen, Carlsbad, CA) was conducted to transfected cells according to the manufacturer's protocol. Overexpressed adenovirus of $\beta$-catenin (Ad- $\beta$-catenin) and adenovirus of control (Ad-con) was constructed by Genechem Co., Ltd (Genechem, Shanghai, China).

\section{Transwell invasion assay:}

Resuspension of H520 cells with $100 \mu \mathrm{l}$ serum-free DMEM were plated in 24-well transwell chamber (Corning, CA, USA) with a Matrigel coated membrane (BD Bioscience, San Jose, USA) for the transwell invasion assay. Lower chambers of the inserts were filled with DMEM medium containing $10 \%$ FBS. 24-well chambers were incubated in a humidified incubator at $37^{\circ}$ with $5 \% \mathrm{CO}_{2} .24 \mathrm{~h}$ later, cells invaded to the lower surface of the insert were fixed, stained and counted under a light microscope.

\section{Cell counting kit-8 (CCK-8) assays:}

The lung cancer cells were cultured in 24-well plate with or without miR-340-5p mimic and then exposed to Ad- $\beta$-catenin for 48 h. $30 \mu \mathrm{l}$ of the CCK-8 (Dojindo, Japan) was added in each well and then cultured at $37^{\circ}$ for $4 \mathrm{~h}$. The absorbance at $450 \mathrm{~nm}$ was detected by microplate by using a microplate reader (Thermo Fisher).

\section{Statistical analysis:}

All data analysis was conducted using the statistical package for the social sciences (SPSS) software package (version 22.0). All the results were shown as mean \pm standard deviation (SD). The significant differences between different groups were analyzed by t-test (comparison for two groups), $\mathrm{p}<0.05$ was considered to be statistically significant.

\section{RESULTS AND DISCUSSION}

MiR-340-5p is down-regulated in lung cancer tissues and cell lines. To determine the expression of miR-340$5 \mathrm{p}$ in lung cancer tissues and cell lines, RT-PCR was conducted to assess miR-340-5p expression in the 38 lung cancer tissues and adjacent normal tissues (fig. 1A). Moreover, the messenger RNA (mRNA) expression of miR-340-5p was also examined in 16HBE and lung cancer cell lines (SKMES1, A549, H23, SPC-A1 and H520 cells) (fig. 1B).

We found that the expression of miR-340-5p in lung cancer tissues was significantly lower than that in adjacent normal tissues. The expression of miR-340-5p in lung cancer cell lines was also significantly lower than that in 16HBE cells and the expression of miR$340-5 p$ decreased most obviously in H520 cell lines. Therefore, we used these H520 cell line as research cell model.

Over-expression of miR-340-5p inhibits lung cancer cell invasion and proliferation. Cancer cell invasion and proliferation are two critical factors for tumor progression and metastasis. To explore whether miR$340-5$ p has a potential role in suppressing lung cancer cell invasion and proliferation, we examined lung cancer H520 cell invasion by using Transwell invasion assay and proliferation by CCK- 8 assay. The number of invaded H520 cells and proliferation were decreased after transfection with miR-340-5p mimic as compared with the control (mimic NC) (fig. 2A-2C). These results demonstrated that the overexpression of miR-340-5p suppresses the invasion and proliferation of lung cancer cells.

$\beta$-catenin and miR-340-5p are negatively correlated in lung cancer tissues and can be negatively regulated by miR-340-5p. Previous studies have shown that miR$340-5 p$ could regulate $\beta$-catenin expression in other cancer diseases $^{[11]}$. In order to clarify whether $\beta$-catenin can be regulated by miR-340-5p in lung cancer cells, we used PCR analysis to detect the $\beta$-catenin expression after treatment with miR-340-5p mimic or inhibitor. The results showed that $\beta$-catenin could be negatively regulated by miR-340-5p (fig. 3A). Moreover, we also found that $\beta$-catenin and miR-340-5p are negatively related in lung cancer tissues (fig. 3B).

$\beta$-catenin pathway mediates the inhibitory effect of miR$340-5 p$ on lung cancer cell invasion and proliferation. To further verify whether $\beta$-catenin pathway is involved in the inhibitory role of miR-340-5p in lung cancer cells, we overexpressed $\beta$-catenin and investigated whether $\beta$-catenin could block the inhibitory role of miR-340$5 \mathrm{p}$ on H520 cell invasion and proliferation (fig. 4A). The CCK- 8 and Transwell assay showed that $\beta$-catenin could significantly reverse the suppressor effect of miR-340-5p on lung cancer proliferation and invasion (fig. 4B and fig. 4C). 
A

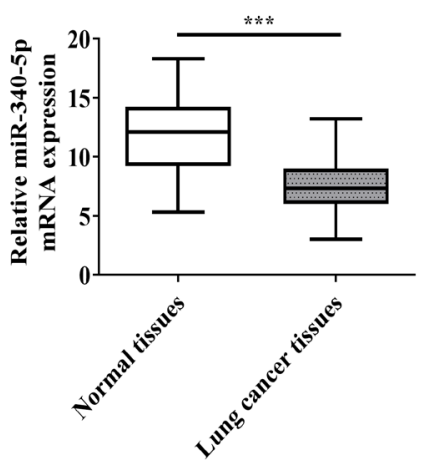

B

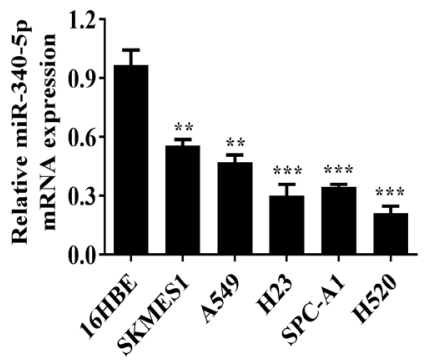

Fig. 1: miR-340-5p is down-regulated in lung cancer tissues and cell lines

(A) RT-PCR analysis was performed to examine the expression of miR-340-5p in the 38 lung cancer tissues and adjacent normal tissues; (B) RT-PCR analysis was used to determine the miR-340-5p expression in lung cancer lines. Each experiment was independently repeated three times; $* * \mathbf{p}<0.01, * * * \mathbf{p}<0.001$
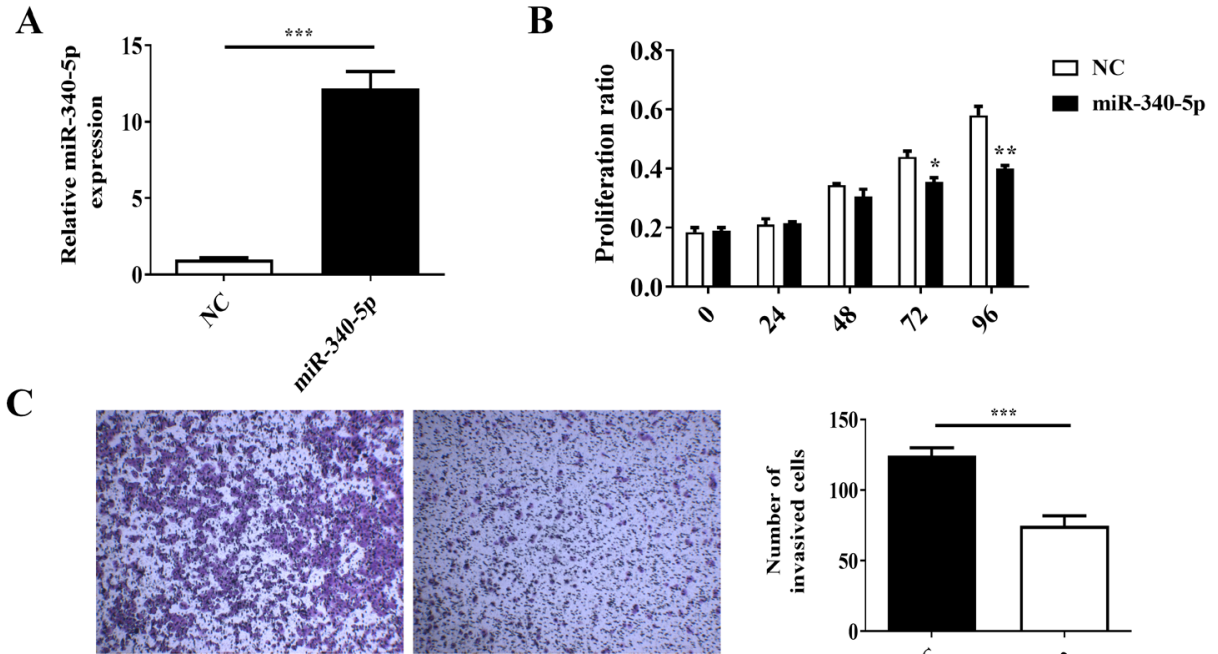

NC

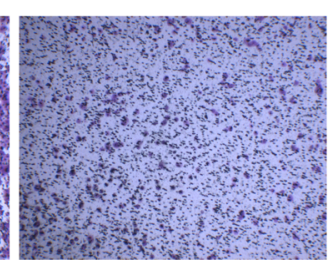

miR-340-5p

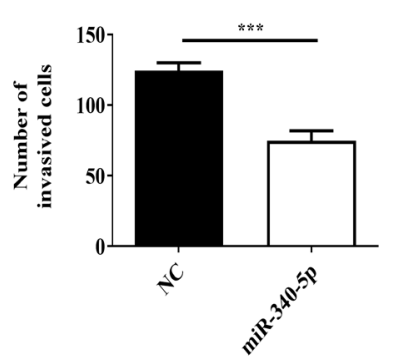

Fig. 2: The effect of miR-340-5p on lung cancer cell invasion and proliferation

(A) PCR analysis was used to detect the transfection efficiency of miR-340-5p mimic; (B) CCK-8 assay was conducted to examine the proliferation of $\mathbf{H 5 2 0}$ cells; (C) Transwell assay was used to detect the invasion of $\mathbf{H 5 2 0}$ cells. Each experiment was independently repeated three times; $* * \mathbf{p}<0.01, * * * \mathbf{p}<0.001$

A

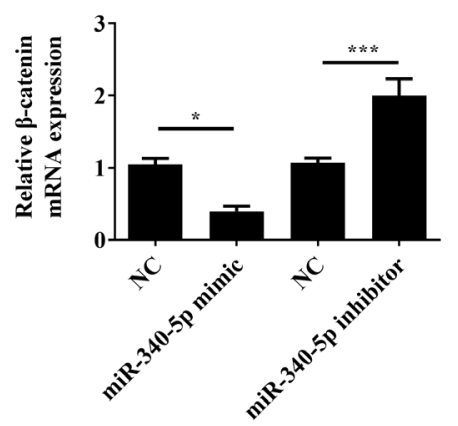

B

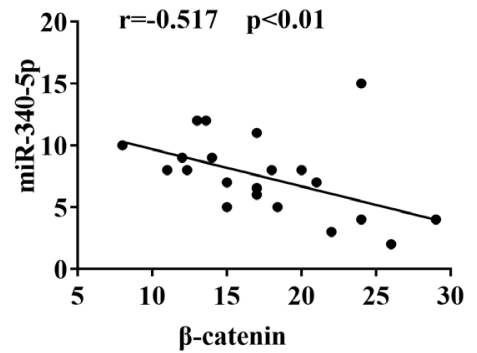

Fig. 3: $\beta$-catenin and miR-340-5p are negatively correlated in lung cancer tissues and can be negatively regulated by miR-340-5p (A) $\beta$-catenin was negatively regulated by miR-340-5p in $\mathrm{H} 520$ cells by PCR analysis; (B) $\beta$-catenin and miR-340-5p are negatively correlated in lung cancer tissues by $\mathrm{PCR}$ analysis. Each experiment was independently repeated three times; ${ }^{*} \mathbf{p}<0.05, * * * p<0.001$ 
A

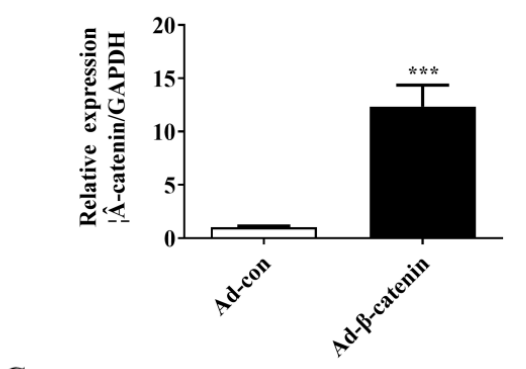

C

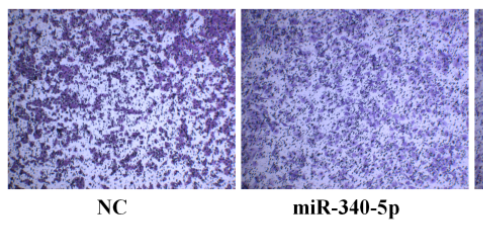

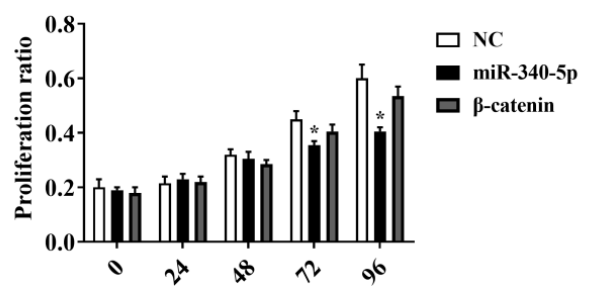

Fig. 4: $\beta$-catenin pathway mediates the inhibitory effect of miR-340-5p in lung cancer cells

(A) PCR analysis was used to confirm the transfection efficiency of adenovirus $\beta$-catenin; (B) CCK-8 assay was conducted to examine the proliferation of $\mathrm{H520}$ cells; (C) Transwell assay was used to detect the invasion of $\mathrm{H} 520$ cells. Each experiment was independently repeated three times; $* * \mathbf{p}<0.01, * * * \mathbf{p}<0.001$

Accumulating evidences have suggested that the pathogenesis and progression of lung cancer is a complex biology process, which is attributed to dysregulation of numerous oncogenes and tumorsuppressive genes ${ }^{[13,14]}$. Although the latest diagnostic and therapeutic techniques have improved the clinical outcomes of patients with lung cancer patients, the prognosis of many lung cancer patients is still very poor $^{[15]}$. Moreover, there are few reliable markers available to accurately predict early stage lung cancer patients, the diagnosis and treatment of lung cancer still face severe challenges ${ }^{[16]}$.

In recent years, scholars have confirmed that miR-340$5 \mathrm{p}$ is down-regulated and involved in the occurrence and development in many types of malignant tumors ${ }^{[11]}$. For example, Rongxin et al. reported that in osteosarcoma, miR-340-5p is down-regulated and can attenuate osteosarcoma development via inhibiting the $\mathrm{Wnt} / \beta$ catenin pathway ${ }^{[11]}$.

Shi et al. demonstrated that miR-340-5p suppresses cell progression and drug resistance in breast cancer via $\mathrm{Wnt} / \beta$-catenin pathway ${ }^{[17]}$. In our present study, we confirmed that miR-340-5p is down regulated in lung cancer tissues and overexpression of miR-340-5p could inhibit lung cancer cell invasion and proliferation in vitro. These results suggest that miR-340-5p may play an important role in lung cancer progression.

No research on the role and underlying mechanism of miR-340-5p in lung cancer has been conducted to date.
In the process of investigating the potential mechanisms by which miR-340-5p inhibiting tumor invasion and proliferation in lung cancer, $\beta$-catenin pathway could promote tumor metastasis and further worsen the condition of many cancer patients ${ }^{[18,19]}$.

Moreover, $\beta$-catenin could be regulated by miR-340-5p in different tumor disease ${ }^{[11]}$. Nevertheless, it is unclear whether miR-340-5p plays its action in lung cancer by regulating $\beta$-catenin pathway. In present study, we found that miR-340-5p could negatively regulate $\beta$-catenin expression to exert its inhibition action on invasion and proliferation in H520 cells. Based on these, our data suggest that up-regulated expression of miR-340$5 \mathrm{p}$ inhibits lung cancer cells invasion by decreasing $\beta$-catenin expression and may have a therapeutic effect in lung cancer.

In conclusion, our results show that miR-340-5p functions as a tumor suppressor gene that attenuates invasion and proliferation in lung cancer by negatively regulating the $\beta$-catenin expression. The data will provide new insights for the treatment of lung cancer and new guidance for clinical treatment of lung cancer.

\section{Acknowledgements:}

This work was supported by The Second Affiliated Hospital of Fujian Medical University.

\section{Conflicts of interest:}

The authors report no conflicts of interest. 


\section{REFERENCES}

1. Unsal M, Kutlar G, Sullu Y, Yurtlu S. Tonsillar metastasis of small cell lung carcinoma. Clin Respir J 2016;10(6):681-3.

2. Jin X, Che DB, Zhang ZH, Yan HM, Jia ZY, Jia XB. Ginseng consumption and risk of cancer: a meta-analysis. J Ginseng Res 2016;40(3):269-77.

3. Yilmaz U, Wong TZ, Marks LB. How good is positron emission tomography at detecting previously-occult metastatic lung cancer at diagnosis? Int J Radiat Oncol Biol Phys 2018;102(4):716-7.

4. Li X, Li M, Chen D, Shi G, Zhao H. PAQR3 inhibits proliferation via suppressing PI3K/AKT signaling pathway in non-small cell lung cancer. Arch Med Sci 2018;14(6):1289-97.

5. Wang W, Wu S, Guo M, He J. LMO4 is a prognostic marker involved in cell migration and invasion in non-small-cell lung cancer. J Thorac Dis 2016;8(12):3682-90.

6. Pan JY, Zhang F, Sun CC, Li SJ, Li G, Gong FY, et al. miR134: a human cancer suppressor?. Mol Ther Nucleic Acids 2017;6:140-9.

7. Li H, Jiang M, Cui M, Feng G, Dong J, Li Y, et al. MiR-365 enhances the radiosensitivity of non-small cell lung cancer cells through targeting CDC25A. Biochem Biophys Res Commun 2019;512(2):392-8.

8. Meng X, Li Z, Zhou S, Xiao S, Yu P. miR-194 suppresses high glucose-induced non-small cell lung cancer cell progression by targeting NFAT5. Thorac Cancer 2019;10(5):1051-9.

9. Cao B, Tan S, Tang H, Chen Y, Shu P. miR-512-5p suppresses proliferation, migration and invasion, and induces apoptosis in non-small cell lung cancer cells by targeting ETS1. Mol Med Rep 2019;19(5):3604-14.

10. Wang R, Chen XF, Shu YQ. Prediction of non-small cell lung cancer metastasis-associated microRNAs using bioinformatics. Am J Cancer Res 2015;5(1):32.

11. Rongxin S, Pengfei L, Li S, Xiaochen J, Yihe H. MicroRNA340-5p suppresses osteosarcoma development by downregulating the $\mathrm{Wnt} / \beta$-catenin signaling pathway via targeting the STAT3 gene. Eur Rev Med Pharmacol Sci 2019;23(3):982-91.
12. Yang L, Men WL, Yan KM, Tie J, Nie YZ, Xiao HJ. MiR$340-5 \mathrm{p}$ is a potential prognostic indicator of colorectal cancer and modulates ANXA3. Eur Rev Med Pharmacol Sci 2018;22(15):4837-45.

13. Nemer G, Khalil A. A cautious note on thalidomide usage in cancer treatment: genetic profiling of the TBX2 sub-family gene expression is required. Drug Res 2019;69(9):512-8.

14. Wang D, Luo Y, Shen D, Yang L, Liu HY, Che YQ. Clinical features and treatment of patients with lung adenocarcinoma with bone marrow metastasis. Tumori J 2019;105(5):388-93.

15. Lee D, Kim S, Palta J, Lewis B, Keall P, Kim T. A retrospective 4D-MRI based on 2D diaphragm profiles for lung cancer patients. J Med Imaging Radiat Oncol 2019;63(3):360-9.

16. Qin KR, Dua D. Diagnostic dilemma: Primary peritoneal mesothelioma with para-occupational asbestos exposure. J Glob Oncol 2017;3(6):828-32.

17. Shi S, Chen X, Liu H, Yu K, Bao Y, Chai J, et al. LGR5 acts as a target of miR-340-5p in the suppression of cell progression and drug resistance in breast cancer via $\mathrm{Wnt} / \beta$-catenin pathway. Gene 2019;683:47-53.

18. Sun $\mathrm{H}$, Jiang $\mathrm{C}$, Cong $\mathrm{L}, \mathrm{Wu} \mathrm{N}$, Wang $\mathrm{X}$, Hao $\mathrm{M}$, et al. CYP24A1 Inhibition facilitates the antiproliferative effect of 1 , $25(\mathrm{OH}) 2 \mathrm{D} 3$ through downregulation of the WNT/ $\beta$-Catenin pathway and methylation-mediated regulation of CYP24A1 in colorectal cancer cells. DNA Cell Biol 2018;37(9):742-9.

19. Yang Z, Ji L, Jiang G, Liu R, Liu Z, Yang Y, et al. FL118, a novel camptothecin analogue, suppressed migration and invasion of human breast cancer cells by inhibiting epithelialmesenchymal transition via the $\mathrm{Wnt} / \beta$-catenin signaling pathway. Biosci Trends 2018;12(1):40-6.

This is an open access article distributed under the terms of the Creative Commons Attribution-NonCommercial-ShareAlike 3.0 License, which allows others to remix, tweak, and build upon the work non-commercially, as long as the author is credited and the new creations are licensed under the identical terms

This article was originally published in a special issue,

"Therapeutic Perspectives in Biomedical Research and Pharmaceutical Sciences and their Nursing Methods"

Indian J Pharm Sci 2021:83(4)Spl issue "133-138" 